\title{
Experimental and theoretical cross sections for positron collisions with 3-hydroxy-tetrahydrofuran
}

\author{
Luca Chiari, ${ }^{1,2, a)}$ Prasanga Palihawadana, ${ }^{2}$ J. R. Machacek, ${ }^{2}$ Casten Makochekanwa, ${ }^{2}$ \\ Gustavo García, ${ }^{3,4}$ Francisco Blanco, ${ }^{5}$ R. P. McEachran, ${ }^{2}$ M. J. Brunger, ${ }^{1,6}$ \\ Stephen J. Buckman, ${ }^{2,6}$ and James P. Sullivan ${ }^{2}$ \\ ${ }^{1}$ ARC Centre for Antimatter-Matter Studies, School of Chemical and Physical Sciences, Flinders University, \\ GPO Box 2100, Adelaide, SA 5001, Australia \\ ${ }^{2}$ ARC Centre for Antimatter-Matter Studies, Research School of Physics and Engineering, The Australian \\ National University, Canberra, ACT 0200, Australia \\ ${ }^{3}$ Instituto de Física Fundamental, Consejo Superior de Investigationes Científicas (CSIC), Serrano 113-bis, \\ 28006 Madrid, Spain \\ ${ }^{4}$ Centre for Medical Radiation Physics, University of Wollongong, Wollongong, NSW 2522, Australia \\ ${ }^{5}$ Departamento de Física Atómica, Molecular y Nuclear, Universidad Complutense de Madrid, \\ 28040 Madrid, Spain \\ ${ }^{6}$ Institute of Mathematical Sciences, University of Malaya, 50603 Kuala Lumpur, Malaysia
}

(Received 20 December 2012; accepted 24 January 2013; published online 15 February 2013)

\begin{abstract}
Cross section results from a joint experimental and theoretical investigation into positron scattering from 3-hydroxy-tetrahydrofuran (3H-THF) are presented. Total and positronium (Ps) formation cross sections have been measured from 1 to $190 \mathrm{eV}$ using the positron beamline at the Australian National University, which has an energy resolution between 60 and $100 \mathrm{meV}$. The total cross section (TCS) and the elastic and total inelastic integral cross sections in the energy range between 1 and $1000 \mathrm{eV}$ have been computed within the Independent Atom Model using the Screening Corrected Additivity Rule approach. In addition, we have calculated elastic differential cross sections at selected incident energies. Our computations represent the first theoretical results reported for this target species, while our measured Ps formation cross sections are also novel. Comparison of the present TCS with the previous results from the University of Trento shows a good level of agreement at the lowest energies. We also provide a comparison between the present cross sections for $3 \mathrm{H}-\mathrm{THF}$ and those from our earlier study on the parent molecule tetrahydrofuran. () 2013 American Institute of Physics. [http://dx.doi.org/10.1063/1.4790620]
\end{abstract}

\section{INTRODUCTION}

Since the pioneering paper of Boudaiffa et al. ${ }^{1}$ the atomic and molecular physics (AMP) and the medical science community have conducted extensive work to investigate the effect of radiation damage in biomolecular systems (see, for instance, the recent book by García Gómez-Tejedor and Fuss ${ }^{2}$ for a state-of-the-art picture of the research carried out in this field). Thus, it is clear why the interactions between positrons, and those biomolecules that make up living matter, have become an important field of study. Unfortunately, due to the practical difficulties in producing a molecular beam or target cell of a pure nucleic acid (note that most of the nucleobases are solid compounds at room temperature), it is not always possible to directly study the biomolecules of interest. Therefore, the AMP community has, to date, focussed its interest on those molecules that can be considered as proto-types, or analogues, for the "building blocks" of the nucleic acids. There is a general agreement among the members of this community $^{3-6}$ upon the reductionist philosophy whereby the physical, chemical, and biological properties of a system stem from the fundamental properties of its constituents and their

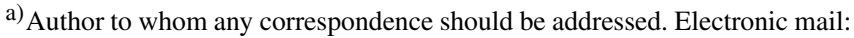
luca.chiari@flinders.edu.au.
}

interactions. ${ }^{7,8}$ The subject of the present study, namely 3hydroxy-tetrahydrofuran (3H-THF: $\mathrm{C}_{4} \mathrm{H}_{8} \mathrm{O}_{2}$ ), is one of those prototypical targets. In fact, similar to its parent molecule tetrahydrofuran ${ }^{9}$ (THF) (see Figure 1), 3H-THF represents an analogue for the sugar rings that constitute the phosphatedeoxyribose backbone structure in the nucleic acids ${ }^{10}$ (see, e.g., Figure 1 in Ref. 11).

Applications to biomedical research are of course not the only motivation for studying these kinds of targets. The investigation of the fundamental forces that drive the scattering process remains of primary interest to the AMP community. In this regard, 3H-THF represents a particularly suitable target. As shown in Figure 1, 3H-THF is a heterocyclic ether similar to THF, except for the hydroxyl $(-\mathrm{OH})$ functional group that lies at position 3 on the five-atom ring. $3 \mathrm{H}-\mathrm{THF}$ also possesses some physico-chemical properties that make it intriguing to study from a more fundamental point of view (see Table I). Therefore, it is not surprising that recent work on $3 \mathrm{H}-\mathrm{THF}^{11-14}$ included fundamental scattering phenomena. Most of those studies, however, have been devoted to investigating this target with electrons as the probe. In particular, there has been the work of Możejko and Sanche ${ }^{12}$ who calculated the elastic integral cross section (ICS) and elastic differential cross sections (DCS) using the Independent Atom Model (IAM) and, later, both Vizcaino et al. ${ }^{13}$ and 

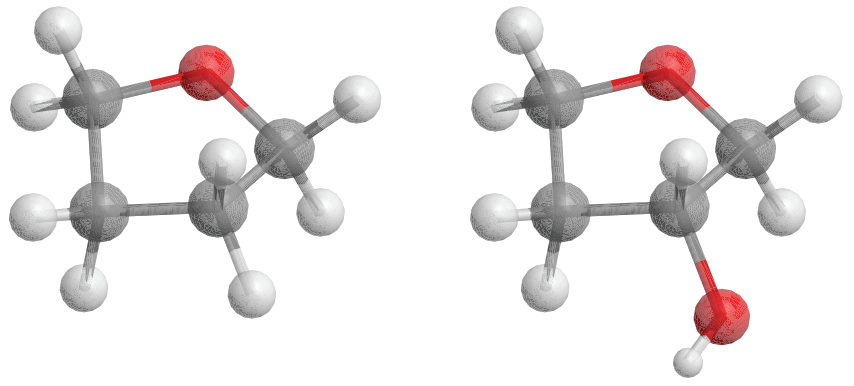

FIG. 1. Schematic diagram of the THF (left) and the 3H-THF (right) molecules. Diagrams were produced using ChemBio3D Ultra. ${ }^{55}$

Milosavljević et al. ${ }^{14}$ reported on measured and calculated elastic ICS and DCS, although they made use of somewhat different theoretical approaches in their investigations. The first study employed the Schwinger multichannel method (SMC), while the second one used the IAM approach with Screening Corrected Additivity Rule (SCAR). The work of Milosavljevic et al. ${ }^{14}$ also included the total cross section (TCS) computed with the same IAM-SCAR method.

Collisions between positrons and $3 \mathrm{H}-\mathrm{THF}$ have not been investigated to the same extent. To date there is one previous experimental study by the University of Trento group, ${ }^{11}$ but to the best of our knowledge there are no calculations. This lack of theory may ensue from the difficulty in constructing a realistic enough physical model for the target, as well as an adequate representation of the interactions involved in the scattering process in its different channels. As far as the measurements are concerned, the presence of only one data set is at least partly due to technical issues. The "sticky" nature of $3 \mathrm{H}-\mathrm{THF}^{11}$ is just one of the experimental challenges that this species offers to conducting scattering measurements. Unlike THF, ${ }^{9,15} 3 \mathrm{H}$-THF at room temperature is not particularly volatile (its vapor pressure is estimated to be just $34 \mathrm{~Pa}$ at $\left.25^{\circ} \mathrm{C}\right),{ }^{16}$ which makes it rather difficult to generate enough vapor $^{11}$ in order to achieve the required target density for a molecular beam in the scattering region. Moreover, $3 \mathrm{H}-\mathrm{THF}$ is a molecule that comes in at least two conformational forms that have quite different physico-chemical properties ${ }^{11}$ (see Table I). On the one hand, this somewhat complicates the interpretation of the experimental results. On the other hand, however, this renders a comparison with the corresponding

TABLE I. Some important physico-chemical properties of the two most energetically stable conformers of THF and 3H-THF: molecular diameter $(D)$, permanent dipole moment $(\mu)$, dipole polarizability $(\alpha)$, first adiabatic ionization energy $\left(E_{\mathrm{i}}\right)$ and positronium formation threshold energy $\left(E_{\mathrm{Ps}}\right)$. Note that $E_{\mathrm{Ps}}=E_{\mathrm{i}}-6.8 \mathrm{eV}$.

\begin{tabular}{lccccc}
\hline \hline Species & $D(\AA)$ & $\mu(\mathrm{D})$ & $\alpha(\mathrm{au})$ & $E_{\mathrm{i}}(\mathrm{eV})$ & $E_{\mathrm{Ps}}(\mathrm{eV})$ \\
\hline THF, 1st and 2nd conformer & $4.63^{\mathrm{a}}$ & $1.63^{\mathrm{b}}$ & $47.08^{\mathrm{c}}$ & $9.57^{\mathrm{d}}$ & 2.77 \\
3H-THF, 1st conformer & $\geq 4.63^{\mathrm{c}}$ & $1.74^{\mathrm{c}}$ & $50.68^{\mathrm{c}}$ & $9.48^{\mathrm{e}}$ & 2.68 \\
3H-THF, 2nd conformer & & $2.88^{\mathrm{c}}$ & $50.98^{\mathrm{c}}$ & & \\
\hline \hline
\end{tabular}

${ }^{\mathrm{a}}$ Reference 24.

${ }^{\mathrm{b}}$ Reference 5 .

${ }^{\mathrm{c}}$ Reference 11

${ }^{\mathrm{d}}$ Reference 54

${ }^{\mathrm{e}}$ Reference 27 cross sections for THF rather intriguing. In fact, as the lowest conformers of THF have largely identical physico-chemical properties to those of the $1 \mathrm{st} 3 \mathrm{H}-\mathrm{THF}$ conformer, this in principle enables us to infer some information on the role that the various conformers of 3H-THF might play in the scattering dynamics. Finally, we also note that $3 \mathrm{H}-\mathrm{THF}$ is a chiral molecule, as, in general, it comes in its left- and right-handed enantiomers, namely $(\mathrm{S})-(+)-3$-hydroxy-tetrahydrofuran and (R)-(-)-3-hydroxy-tetrahydrofuran. The possible importance of chirality in both nature and in positron scattering measurements was recently examined by Chiari et al. ${ }^{17}$ to whom the reader is referred for more details.

In this paper, we report on new TCS and positronium (Ps) formation cross sections for positron impact with $3 \mathrm{H}$ THF, between 1 and $190 \mathrm{eV}$, that were measured using the positron beamline at the Australian National University. ${ }^{18} \mathrm{We}$ also present the first theoretical results for positron scattering from $3 \mathrm{H}-\mathrm{THF}$ including the TCS, the elastic ICS, the summed inelastic ICS (excluding rotations and vibrations) and some elastic DCS calculated using the IAM-SCAR method at selected energies in the energy range from 1 to $1000 \mathrm{eV}$. We compare our measured and calculated results for $3 \mathrm{H}-\mathrm{THF}$ with those from our recent investigation on $\mathrm{THF},{ }^{9}$ in order to observe how small changes in the molecular structure (which may lead to quite different molecular properties) can affect the scattering process for those systems. Studies of positronmolecule collisions, in which TCS for a series of structurally related species are investigated, are actually quite rare. Note that in organic chemistry, structurally related molecules are often studied to better understand corresponding trends in their chemical activity. The review by Kimura et al.; ${ }^{19}$ the work of Zecca et al. ${ }^{20}$ on benzene, cyclohexane, and aniline; and that of Chiari et al. ${ }^{21}$ on biologically relevant molecules are examples of this kind of work.

In Sec. II of this paper we present the experimental procedures of our measurements, while in Sec. III we introduce the theoretical formalism for our calculations. The current results, and a discussion of those outcomes, are then reported in Sec. IV, with some concluding remarks being drawn at the end.

\section{EXPERIMENTAL DETAILS}

The present measurements were carried out at the Australian Positron Beamline Facility. ${ }^{18}$ This apparatus is based on a positron trap and pulsed beam system, ${ }^{22}$ and details of its operation can be found in a recent paper. ${ }^{9}$ We simply recall here that low-energy positrons are obtained from a ${ }^{22} \mathrm{Na}$ radioactive source (activity of $\sim 40 \mathrm{mCi}$ ) in conjunction with a solid neon moderator. The beam is then radially confined by uniform solenoidal magnetic fields (up to $530 \mathrm{G}$ ) which are present throughout the rest of the beamline. The positrons are first transported into a two-stage buffer-gas trap, where the associated electrodes form a stepped electrostatic-potential-well structure, which traps positrons that lose energy through inelastic collisions with a mixture of $\mathrm{N}_{2}$ and $\mathrm{CF}_{4}$ buffer gases. This results in a cloud of positrons that have thermalized to the gas (room) temperature and are subsequently used to form a pulsed positron beam. The trap is typically operated at a 
repetition rate of $60-100 \mathrm{~Hz}$ with up to $\sim 1000$ positrons emitted in each pulse. The positrons then travel into a scattering cell where they interact with the target molecules. The scattering cell is made of gold-plated copper and is $20 \mathrm{~cm}$ long, with entrance and exit apertures that are $5 \mathrm{~mm}$ in diameter. The strong magnetic fields present in the scattering cell region, and downstream from it, ensure that all the positrons, except for those that form Ps and annihilate within the cell, reach and subsequently pass through a retarding potential analyzer (RPA). Note that the RPA is sensitive only to the parallel component of the beam energy $\left(E_{\|}\right)$. The positrons transmitted by the RPA are finally detected by a double-stack, micro-channel plate assembly.

In our measurements, the energy at which the positrons collide with the target molecules is defined by the potential of the scattering cell. The zero for the energy scale is established with a retarding potential analysis of the beam and we estimate the uncertainty on the energy scale to be $\pm 25 \mathrm{meV}$. This level of accuracy is not unusual in an RPA device operated in a high magnetic field. The same retarding potential analysis enables us to estimate the energy distribution of the beam. Careful control of the dumping stage of the trap cycle allows the energy width of the beam to be close to the temperature of the trapped positron cloud. For these measurements, the energy resolution of the beam was between 60 and $100 \mathrm{meV}$ (full-width at half-maximum) owing to variations in the beam formation characteristics.

Care needs to be exercised in order to perform accurate scattering cross section measurements. In particular, in order to minimize multiple scattering effects, the target pressure inside the cell is maintained at a value such that the total scattering probability is no more than $10 \%$ of the unscattered beam intensity. In addition, given the several experimental difficulties in handling $3 \mathrm{H}-\mathrm{THF}$, due to its low vapor pressure at room temperature and its "stickiness" (see Sec. I), we had to heat the sample container and the scattering chamber up to $\sim 60{ }^{\circ} \mathrm{C}$, whereas the target gas lines and the needle valve were kept at $\sim 90^{\circ} \mathrm{C}$. As the scattering cell temperature was different from that of the pressure gauge $\left(45^{\circ} \mathrm{C}\right)$, the pressure readings inside the scattering cell were corrected for the thermal transpiration effect. This was accomplished using the model of Takaishi and Sensui, ${ }^{23}$ and the correction resulted in an increase in the magnitude of the measured cross sections of $\sim 2.3 \%$. The value of the $3 \mathrm{H}-\mathrm{THF}$ molecular diameter that we used in this calculation was $4.63 \AA$, which is actually the known hard-sphere diameter of THF. ${ }^{24}$ Nevertheless, it has been argued that quantum-chemical geometry calculations for these two molecules yield results that justify this assumption. ${ }^{11}$ We finally note that we used a high-purity (99\%) 3H-THF sample (Aldrich) throughout the present measurements. As our sample is a racemate, the proportion of the two $3 \mathrm{H}$-THF enantiomers in our target sample is expected to be approximately the same.

In all linear transmission scattering experiments the basic principle behind the TCS measurements is the Beer-Lambert law. It allows one to derive the TCS from a knowledge of attenuation measurements of the beam intensity, the target pressure in the scattering cell and the length of the interaction region. The methods used in the present experiment to measure the TCS and the Ps formation cross sections are based on the principles of positron motion and scattering in a strong magnetic field ${ }^{25}$ and have been previously presented, as well as our data analysis techniques. ${ }^{26}$ Briefly, the cross sections are determined by measuring specific fractions of the positron beam transmitted through the RPA with the target vapor present in the scattering cell. In a collision with a target molecule, the positron can be elastically scattered through some angle $\theta$ and lose some of its $E_{\|}$in the process. It can also lose some of its total energy if inelastic processes, such as electronic excitations or direct ionization, are energetically allowed. As the RPA discriminates against $E_{\|}$only, a retarding potential analysis provides a simple measurement of the total scattering. Ps formation is also possible above the Ps formation threshold $\left(E_{\mathrm{Ps}}\right)$. However, this process manifests as a loss of positron intensity in the RPA transmission curve, and is easily accounted for. The first adiabatic ionization energy $\left(E_{\mathrm{i}}\right)$ of the most stable $3 \mathrm{H}-\mathrm{THF}$ conformer is $9.48 \mathrm{eV},{ }^{27}$ and given $E_{\mathrm{Ps}}=E_{\mathrm{i}}-6.8 \mathrm{eV}$, we have $E_{\mathrm{Ps}}=2.68 \mathrm{eV}$ for $3 \mathrm{H}-\mathrm{THF}$ (see also Table I).

As with any scattering-cell based experiment, our measured TCS are inevitably affected by the forward angle scattering effect. ${ }^{28}$ As we have extensively covered this issue in a dedicated paper $^{28}$ and we have revisited those details in our most recent study, ${ }^{9}$ we only recall here that this effect causes our measured TCS to be underestimated with respect to their "true" value. This effect can be corrected for, provided that the angular discrimination of the spectrometer and the elastic DCS for the target species of interest are known at any given energy. ${ }^{28}$ This can be achieved, for instance, by following the procedure outlined by Hamada and Sueoka. ${ }^{29}$ The missing angular ranges $0^{\circ} \pm \theta_{\min }$ and $180^{\circ} \pm \theta_{\min }$, where $\theta_{\min }$ is the angular discrimination, can be calculated, for instance, using Eq. (1) in Ref. 9. In doing so, we estimate the angular discrimination in our measurements to vary from $\sim 23^{\circ}$ at $1 \mathrm{eV}$ to $\sim 1.8^{\circ}$ at $150 \mathrm{eV}$, as listed in Table II. The elastic DCS for positron scattering from $3 \mathrm{H}-\mathrm{THF}$ are available from our IAMSCAR computations (see Sec. III, as well as Figure 2 and Table III). However, given that there are neither experimental nor independent theoretical validations of our calculated DCS, employing those DCS to correct our measured TCS might be somewhat premature. As a consequence, in general,

TABLE II. Estimates of the missing angular range $\left(0^{\circ} \pm \theta_{\min }\right)$ in the present measured TCS, and of the corresponding correction for the forward angle scattering effect. That latter correction was calculated with the present IAMSCAR elastic DCS, including the Born dipole rotational excitations (see Table III), at selected positron scattering energies.

\begin{tabular}{lcc}
\hline \hline Energy $(\mathrm{eV})$ & $\theta_{\min }(\mathrm{deg})$ & Correction $(\%)$ \\
\hline 1 & 23 & 41 \\
2 & 16 & 33 \\
5 & 10 & 23 \\
10 & 7.0 & 21 \\
20 & 5.0 & 15 \\
50 & 3.1 & 8.6 \\
100 & 2.2 & 6.6 \\
150 & 1.8 & 4.1 \\
\hline \hline
\end{tabular}




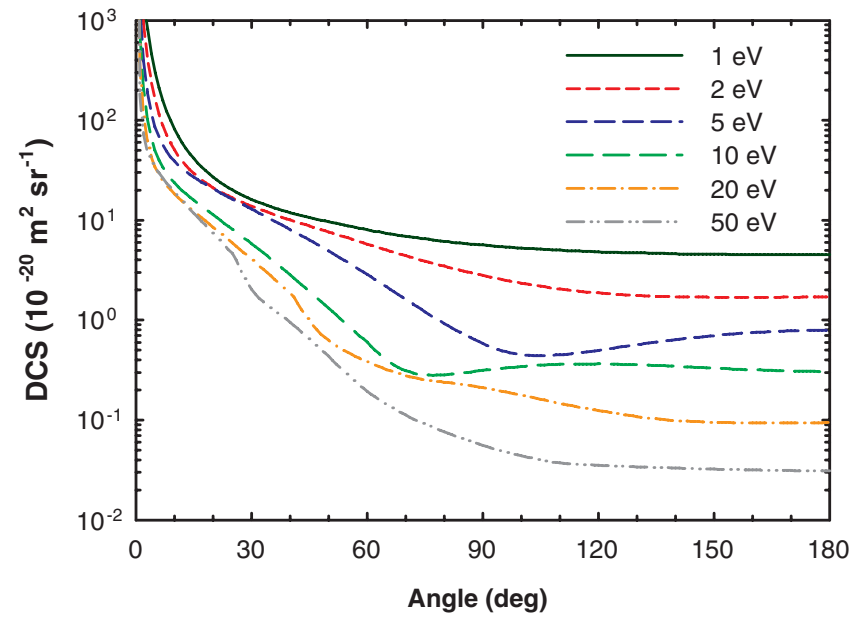

FIG. 2. The present elastic DCS for positron collisions with 3H-THF, as calculated with our IAM-SCAR approach with Born dipole rotational excitations, at selected incident energies. See also the legend in the figure.

we have not done so and the TCS that we present in Table IV and Figures 3 and 5 represent a lower bound on their "real" values. Nevertheless, in order to estimate the extent of the forward angle scattering effect, we have employed our elastic DCS to calculate the correction to our measured TCS at a few selected positron energies (see Table II and Figure 3). In doing so, we find that the magnitude of the TCS we list in Table IV would increase between $\sim 41 \%$ at $1 \mathrm{eV}$ and $\sim 4.1 \%$ at $150 \mathrm{eV}$. Hence, similar to what we found in our earlier investigations on polar polyatomic molecules, ${ }^{9,30}$ we can expect the forward angle scattering effect to play an important role at the lower energies and become progressively less significant towards the higher energies.
The statistical uncertainties on the measured TCS are in the range $2 \%-5 \%$, while those on the Ps formation cross section lie, on average, at around $15 \%$, although they can be significantly larger under some circumstances. Systematic errors in our measurements (e.g., the drift in the absolute pressure scale and the uncertainty on the thermal transpiration correction) have been accounted for and estimated, as discussed in an earlier paper. ${ }^{26}$ The overall uncertainties are calculated as the square root of the quadratic sum of the individual contributing errors. The absolute uncertainties on our measured TCS range from $\sim 3 \%$ to $\sim 7 \%$, whereas those on the Ps formation cross sections are generally within the range $\sim 12 \%-25 \%$.

\section{THEORY DETAILS}

The IAM approach within the SCAR formalism has been extensively used for modeling electron collisions with a large variety of different sized molecules, ${ }^{31-34}$ over a broad energy range, typically between 1 and $5000 \mathrm{eV}$. More recently, the IAM-SCAR method has also been successfully applied to positron scattering studies from argon, ${ }^{35}$ diatomics such as molecular oxygen ${ }^{36}$ and to macro-molecules, such as THF, ${ }^{9}$ where fair agreement was typically found with the reported measurements. As the details of our theory have already been presented in those papers, here we only briefly summarize the method behind our calculations.

\section{A. Atomic optical model}

The first aspects of our computations are the individual atoms constituting the target molecule, that is $\mathrm{C}, \mathrm{H}$, and $\mathrm{O}$ in this case. Our approach is based on an optical model in which

TABLE III. The present elastic DCS calculated with our IAM-SCAR approach, with the Born dipole rotational excitations, at selected scattering energies.

\begin{tabular}{lcccccc}
\hline \hline & \multicolumn{5}{c}{$\mathrm{DCS}\left(10^{-20} \mathrm{~m}^{2} \mathrm{sr}^{-1}\right)$} \\
\cline { 2 - 7 } Angle (deg) & $1 \mathrm{eV}$ & $2 \mathrm{eV}$ & $5 \mathrm{eV}$ & $10 \mathrm{eV}$ & $20 \mathrm{eV}$ & $50 \mathrm{eV}$ \\
\hline 0 & $3.53 \times 10^{9}$ & $7.06 \times 10^{9}$ & $1.76 \times 10^{10}$ & $3.53 \times 10^{10}$ & $7.06 \times 10^{10}$ & $1.76 \times 10^{11}$ \\
10 & 81.77 & 50.69 & 38.92 & 23.63 & 18.31 & 19.18 \\
20 & 26.99 & 21.11 & 20.61 & 11.26 & 8.48 & 7.39 \\
30 & 16.13 & 13.78 & 12.88 & 5.80 & 4.06 & 2.04 \\
40 & 11.90 & 10.03 & 8.04 & 2.83 & 1.85 & 0.95 \\
50 & 9.66 & 7.59 & 4.90 & 1.31 & 0.62 & 0.43 \\
60 & 8.01 & 5.74 & 2.86 & 0.60 & 0.38 & 0.19 \\
70 & 6.92 & 4.40 & 1.61 & 0.31 & 0.28 & 0.11 \\
80 & 6.16 & 3.47 & 0.92 & 0.28 & 0.24 & 0.08 \\
90 & 5.66 & 2.79 & 0.58 & 0.31 & 0.21 & 0.06 \\
100 & 5.29 & 2.34 & 0.45 & 0.34 & 0.18 & 0.04 \\
110 & 5.01 & 2.04 & 0.45 & 0.36 & 0.15 & 0.04 \\
120 & 4.84 & 1.86 & 0.50 & 0.37 & 0.12 & 0.04 \\
130 & 4.70 & 1.76 & 0.57 & 0.36 & 0.11 & 0.03 \\
140 & 4.62 & 1.71 & 0.64 & 0.34 & 0.10 & 0.03 \\
150 & 4.56 & 1.70 & 0.70 & 0.33 & 0.09 & 0.03 \\
160 & 4.51 & 1.69 & 0.75 & 0.32 & 0.09 & 0.03 \\
170 & 4.51 & 1.70 & 0.78 & 0.31 & 0.09 & 0.03 \\
180 & 4.51 & 1.70 & 0.79 & 0.31 & 0.09 & 0.03 \\
\hline \hline
\end{tabular}


TABLE IV. The present measured TCS $\left(\sigma_{\mathrm{T}}\right)$ and Ps formation cross sections $\left(\sigma_{\mathrm{Ps}}\right)$, together with their total uncertainties $(\Delta \sigma)$.

\begin{tabular}{|c|c|c|c|c|}
\hline Energy $(\mathrm{eV})$ & $\sigma_{\mathrm{T}}\left(10^{-20} \mathrm{~m}^{2}\right)$ & $\Delta \sigma_{\mathrm{T}}\left(10^{-20} \mathrm{~m}^{2}\right)$ & $\sigma_{\mathrm{Ps}}\left(10^{-20} \mathrm{~m}^{2}\right)$ & $\Delta \sigma_{\mathrm{Ps}}\left(10^{-20} \mathrm{~m}^{2}\right)$ \\
\hline 1.0 & 73.30 & 2.41 & & \\
\hline 1.5 & 61.50 & 2.37 & & \\
\hline 2.0 & 54.78 & 2.22 & & \\
\hline 2.5 & 49.54 & 2.10 & 0.18 & 1.52 \\
\hline 3.0 & 45.59 & 2.06 & 1.43 & 1.46 \\
\hline 3.5 & 44.63 & 2.07 & 3.18 & 1.53 \\
\hline 4.0 & 43.56 & 2.08 & 5.21 & 1.53 \\
\hline 4.5 & 41.20 & 2.10 & 4.10 & 1.54 \\
\hline 5.0 & 41.49 & 1.99 & 6.61 & 1.48 \\
\hline 6.0 & 39.23 & 1.99 & 7.17 & 1.52 \\
\hline 7.0 & 38.81 & 1.97 & 8.44 & 1.51 \\
\hline 8.0 & 38.02 & 1.95 & 9.66 & 1.50 \\
\hline 9.0 & 36.85 & 1.90 & 10.41 & 1.48 \\
\hline 10.0 & 36.93 & 1.87 & 12.29 & 1.43 \\
\hline 11.0 & 36.80 & 1.94 & 11.80 & 1.49 \\
\hline 12.0 & 37.46 & 1.88 & 12.34 & 1.49 \\
\hline 13.0 & 37.11 & 1.92 & 11.53 & 1.43 \\
\hline 14.0 & 34.32 & 1.84 & 11.64 & 1.47 \\
\hline 15.0 & 34.19 & 1.82 & 10.76 & 1.38 \\
\hline 16.0 & 33.39 & 1.82 & 11.84 & 1.47 \\
\hline 17.0 & 34.54 & 1.92 & 10.92 & 1.47 \\
\hline 18.0 & 34.49 & 1.88 & 10.79 & 1.45 \\
\hline 19.0 & 32.48 & 1.83 & 10.13 & 1.41 \\
\hline 20.0 & 32.94 & 1.77 & 10.43 & 1.43 \\
\hline 21.0 & 33.16 & 1.80 & 9.94 & 1.38 \\
\hline 22.0 & 32.03 & 1.81 & 8.50 & 1.40 \\
\hline 23.0 & 34.44 & 1.83 & 10.77 & 1.38 \\
\hline 24.0 & 33.58 & 1.77 & 10.49 & 1.37 \\
\hline 25.0 & 33.40 & 1.78 & 9.12 & 1.36 \\
\hline 26.0 & 31.92 & 1.71 & 8.08 & 1.36 \\
\hline 28.0 & 32.33 & 1.75 & 7.94 & 1.35 \\
\hline 30.0 & 34.09 & 1.70 & 9.21 & 1.27 \\
\hline 32.0 & 31.59 & 1.92 & 8.15 & 1.46 \\
\hline 34.0 & 33.24 & 1.89 & 7.87 & 1.41 \\
\hline 36.0 & 31.61 & 1.85 & 7.68 & 1.37 \\
\hline 38.0 & 33.76 & 1.85 & 8.49 & 1.42 \\
\hline 40.0 & 32.14 & 1.86 & 7.54 & 1.42 \\
\hline 42.0 & 31.58 & 1.86 & 6.41 & 1.38 \\
\hline 44.0 & 31.45 & 1.76 & 5.98 & 1.36 \\
\hline 46.0 & 32.51 & 1.83 & 6.44 & 1.36 \\
\hline 48.0 & 32.71 & 1.82 & 5.22 & 1.34 \\
\hline 50.0 & 31.99 & 1.76 & 6.46 & 1.36 \\
\hline 52.0 & 31.85 & 1.81 & 5.01 & 1.32 \\
\hline 54.0 & 31.57 & 1.77 & 4.88 & 1.36 \\
\hline 56.0 & 31.76 & 1.77 & 5.18 & 1.33 \\
\hline 58.0 & 31.51 & 1.76 & 6.25 & 1.28 \\
\hline 60.0 & 30.27 & 1.67 & 4.65 & 1.19 \\
\hline 70.0 & 31.17 & 2.09 & 5.31 & 1.49 \\
\hline 80.0 & 29.51 & 2.05 & 3.59 & 1.46 \\
\hline 90.0 & 30.84 & 2.06 & 4.52 & 1.41 \\
\hline 100.0 & 31.18 & 2.05 & 5.47 & 1.36 \\
\hline 110.0 & 28.96 & 1.92 & 3.55 & 1.34 \\
\hline 120.0 & 28.73 & 1.95 & 3.74 & 1.34 \\
\hline 130.0 & 27.76 & 1.95 & 2.67 & 1.40 \\
\hline 140.0 & 26.50 & 1.89 & 2.87 & 1.36 \\
\hline 150.0 & 29.17 & 1.95 & 3.55 & 1.42 \\
\hline 160.0 & 26.83 & 1.90 & 3.41 & 1.33 \\
\hline 170.0 & 28.16 & 1.89 & 2.98 & 1.27 \\
\hline 180.0 & 23.44 & 1.72 & 0.63 & 1.15 \\
\hline 190.0 & 24.07 & 1.54 & 1.39 & 0.82 \\
\hline
\end{tabular}



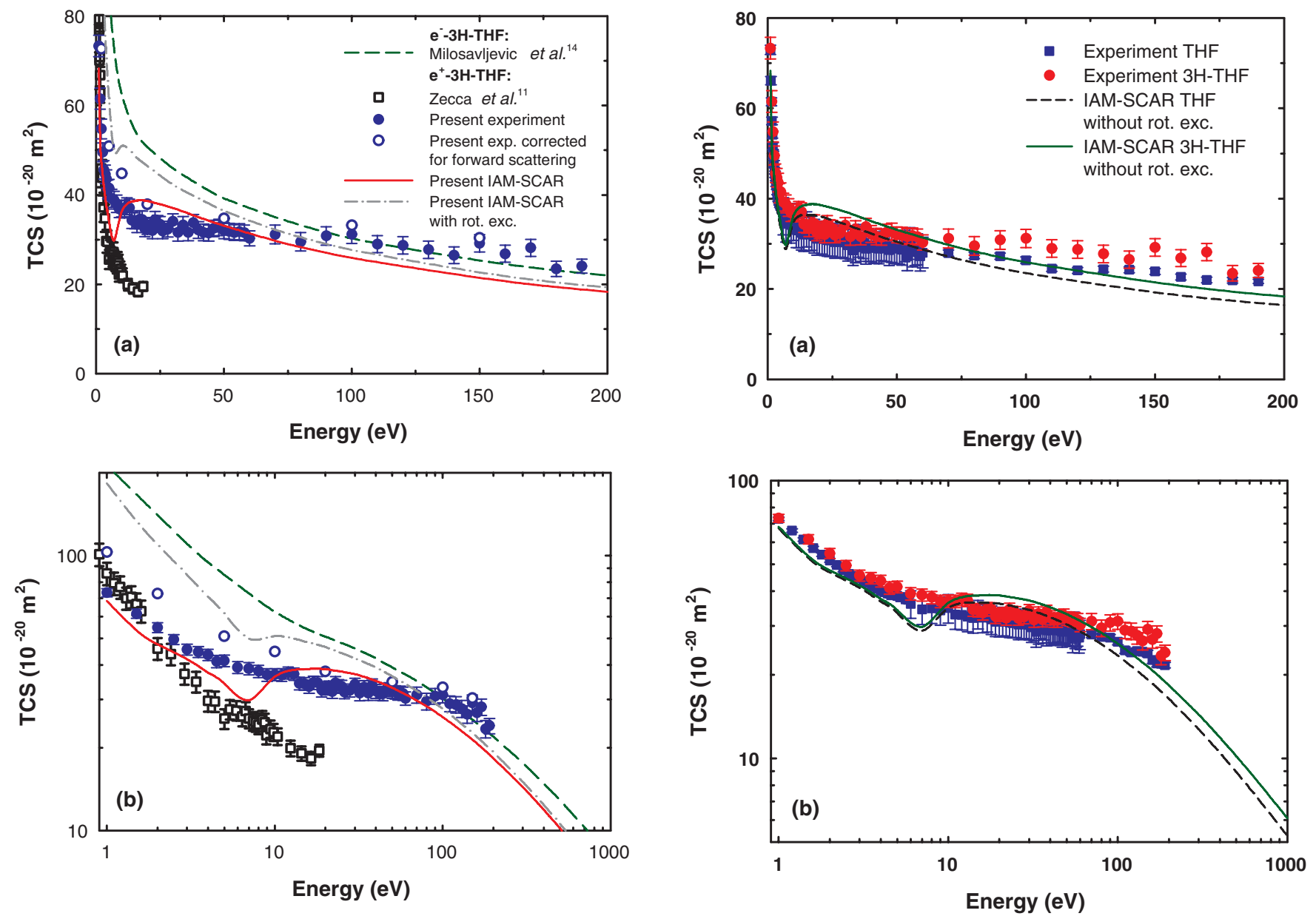

FIG. 3. (a) The present experimental TCS for positron scattering from $3 \mathrm{H}-$ THF is compared to that from Zecca et al. ${ }^{11}$ The present measured TCS corrected for forward angle scattering are also given at selected energies (see Table II). Also plotted are the present TCS calculated with our IAM-SCAR method with and without the Born dipole rotational excitations. See legend in the upper panel for more details. Additionally shown are the electron-impact TCS computed by Milosavljevic et al. ${ }^{14}$ (b) Same as (a) but in log-log scale.

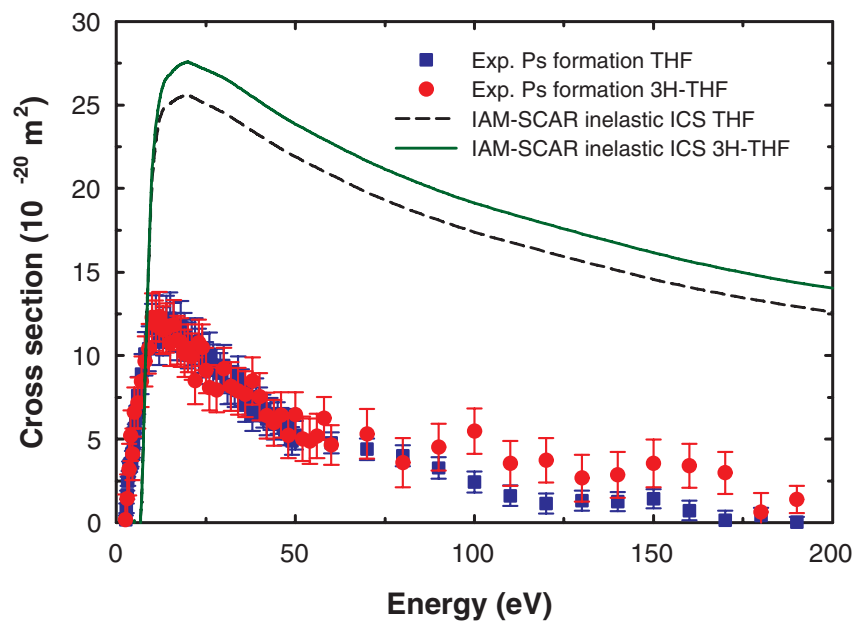

FIG. 4. The present measured Ps formation cross section and IAM-SCAR summed inelastic ICS (except rotations and vibrations) for positron collisions with $3 \mathrm{H}-\mathrm{THF}$. Also shown are the corresponding cross sections from our earlier investigation on positron scattering from THF. ${ }^{9}$
FIG. 5. (a) Comparison between the present measured and calculated (without the Born dipole rotational excitations) TCS for positron collisions with 3 H-THF and our previous results for THF. ${ }^{9}$ (b) Same as (a) but in log-log scale.

the local complex potential is defined as

$$
V(r)=V_{\mathrm{s}}(r)+V_{\mathrm{p}}(r)+\mathrm{i} V_{\mathrm{a}}(r) .
$$

The real part in Eq. (1) represents the elastic scattering process and consists of the electrostatic interaction term, $V_{\mathrm{s}}(r)$, and the polarization term, $V_{\mathrm{p}}(r)$, whereas the imaginary part accounts for inelastic processes which are considered as absorptions, $V_{\mathrm{a}}(r)$, from the incident positron beam. The details of our static, polarization and absorption potentials have recently been described by Chiari et al. ${ }^{36}$ and therefore are not revisited here.

Nevertheless, it is worthwhile to stress that, unlike with electrons, for positron collisions the definition of the energy for the absorption threshold $(\Delta)$ is somewhat controversial. ${ }^{31,32}$ In the case of electron scattering, $\Delta$ would be the excitation energy of the first electronic state of the atom in question. However, for positron collisions, Ps formation is a dominant inelastic scattering channel that generally becomes open at a lower energy than that of the first excited electronic level. As Ps formation cannot be explained in terms of binary collisions, ${ }^{37}$ it cannot be explicitly introduced into the original formulation of the absorption potential as an independent inelastic process. For this reason, Reid and Wadehra ${ }^{38}$ 
suggested the use of the Ps formation threshold energy $\left(\Delta_{\mathrm{p}}\right)$ as the absorption threshold parameter (i.e., $\Delta=\Delta_{\mathrm{p}}$ ). This approach was later shown to somewhat overestimate the TCS at the higher energies. Hence, we define an energy dependent parameter for the absorption threshold $(\Delta(E))$ of the form

$$
\Delta(E)=\Delta-\left(\Delta-\Delta_{\mathrm{p}}\right) e^{-\left(\frac{E-\Delta_{\mathrm{p}}}{E_{\mathrm{m}}}\right)} .
$$

In Eq. (2), $\Delta$ is the lowest excitation energy for the atom and $E_{\mathrm{m}}$ is a characteristic energy at which the absorption potential, without Ps formation, gives the maximum cross section $\left(E_{\mathrm{m}}\right.$ $=20 \mathrm{eV}$ in this case). $E_{\mathrm{m}}$ modulates the negative exponential transition between the limits $\Delta(E)=\Delta_{\mathrm{p}}$ for energies around the Ps formation energy and $\Delta(E)=\Delta$ for higher energies.

The choice of the polarization potential is particularly important for positron scattering calculations, as it is the only attractive contribution to the positron-atom interaction (except for virtual Ps formation). Here we use a new polarization potential based on that developed by McEachran et $a{ }^{39}$ for the noble gases. In particular, we employ the dipole plus quadrupole polarization potentials for $\mathrm{Ne}$ given by McEachran et al., ${ }^{39}$ but scaled by a constant in order to give the known dipole $\left(\alpha_{\mathrm{d}}\right)$ and quadrupole $\left(\alpha_{\mathrm{q}}\right)$ polarizabilities of the $\mathrm{C}\left(\alpha_{\mathrm{d}}=11.88 \mathrm{au}, \alpha_{\mathrm{q}}=54.76 \mathrm{au}\right),{ }^{40,41} \mathrm{H}\left(\alpha_{\mathrm{d}}\right.$ $\left.=4.50 \mathrm{au}, \alpha_{\mathrm{q}}=15 \mathrm{au}\right),{ }^{40,42}$ and $\mathrm{O}\left(\alpha_{\mathrm{d}}=5.41 \mathrm{au}, \alpha_{\mathrm{q}}=16.90\right.$ $\mathrm{au})^{40,41}$ atoms (see Ref. 36 for further details).

\section{B. Screening Corrected Additivity Rule}

In order to calculate the cross sections for positron scattering from the $\mathrm{C}_{4} \mathrm{H}_{8} \mathrm{O}_{2}$ molecule, we then apply the additivity rule (AR) technique to our IAM results for each constituent atom. In this approach, the molecular scattering amplitude is derived from the sum of all the relevant atomic amplitudes, including the phase coefficients, thus leading to the DCS for the molecule in question. ICS can then be determined by integrating those DCS, with the sum of the elastic ICS and absorption ICS (for all inelastic processes except rotations and vibrations) then giving the TCS. However, the AR does not take into account the molecular structure, so that it is really only applicable when the incident particles are so fast that they effectively "see" the target molecule as a sum of the individual atoms (typically above $\sim 100 \mathrm{eV}$ ). In order to reduce this limitation, García and colleagues ${ }^{34,43}$ introduced the SCAR method. This takes the geometry of the relevant molecule (atomic positions and bond lengths) into account by using some screening coefficients. With this correction the range of validity might be extended to impact energies of $30 \mathrm{eV}$ or a little lower, at least as far as electron scattering is concerned. With respect to positron scattering, this lower energy limit still needs further study, particularly at the elastic DCS level.

\section{Rotational excitations}

From the above description of the IAM-SCAR procedure, it is clear that vibrational and rotational excitations are not included in the present calculations. However, for polar molecules such as $3 \mathrm{H}-\mathrm{THF}$, additional dipole-induced exci- tation cross sections can be calculated following the procedure suggested by Jain. ${ }^{44}$ Basically, in this approach, rotational excitation DCS and ICS for a free electric dipole are calculated in the framework of the first Born approximation (FBA), which can be incorporated into our IAM-SCAR calculation in an incoherent way, just by adding their results as an independent channel. Although rotational excitation energies are, in general, very small (typically a few meV) in comparison with the present incident energies, in order to validate the Born approximation the incident energies should be higher than about a few eV. Under these circumstances, rotational excitation cross sections $J \rightarrow J^{\prime}$ were calculated by weighting the population for the $J$ th rotational quantum number at $300 \mathrm{~K}$, and estimating the average excitation energy from the corresponding rotational constants.

In addition, when the permanent dipole moment of the molecule is significantly large, the FBA also fails for middle and large scattering angles. In order to partially overcome this limitation, we introduced a correction based on that suggested by Dickinson, ${ }^{45}$ which brings an improvement for electron and positron scattering cross sections with strongly polar molecules. This procedure introduces a first-order corrective term to the differential cross sections $\left(\mathrm{d} \sigma^{\text {Dck }} / \mathrm{d} \Omega\right.$ ) for the middle and large angles, but maintains the FBA correction $\left(\mathrm{d} \sigma^{\mathrm{B}} /\right.$ $\mathrm{d} \Omega$ ) for the forward scattering angles:

$$
\begin{aligned}
& \frac{\mathrm{d} \sigma^{\mathrm{B}}}{\mathrm{d} \Omega} \approx \frac{\mu^{2}}{6 E} \frac{1}{\sin ^{2}(\theta / 2)} \quad \theta<\theta_{\mathrm{c}}, \\
& \frac{\mathrm{d} \sigma^{\mathrm{Dck}}}{\mathrm{d} \Omega} \approx \frac{\pi \mu}{64 E} \frac{1}{\sin ^{3}(\theta / 2)} \quad \theta>\theta_{\mathrm{c}} .
\end{aligned}
$$

In Eqs. (3) and (4), $\mu$ is the permanent dipole moment of the molecule and $E$ the incident energy. Provided that $\mu>0.75 \mathrm{D}$, both curves smoothly join together at $\theta=\theta_{\mathrm{c}}$, i.e., the critical angle at which they cross each other.

\section{RESULTS AND DISCUSSION}

\section{A. Total cross sections}

We present in Figure 3 our measured and calculated TCS for positron scattering from $3 \mathrm{H}-\mathrm{THF}$ and we also list the corresponding numerical values in Tables IV and V, respectively. The present theoretical results represent the first computed TCS to be reported for this species. We observe in Figure 3 that the general trend of our experimental and theoretical TCS is a marked decrease in magnitude as a function of increasing positron energy. This TCS behavior is predominantly associated with the important role that the large permanent dipole moment and strong dipole polarizability of the target play at low energy in the scattering dynamics. This is not an original observation, as we have previously noticed this same kind of behavior in the low-energy TCS from our earlier studies on polyatomic polar molecules, such as water and formic acid, ${ }^{30}$ and THF. ${ }^{9}$ The independent work carried out in the last decade at the University of Trento (see, e.g., Refs. 11, 15, 17, 20, and 21) also noted similar trends for polar targets. 
TABLE V. The present TCS $\left(\sigma_{\mathrm{T}}\right)$, elastic $\left(\sigma_{\mathrm{E}}\right)$, and summed inelastic ICS $\left(\sigma_{\mathrm{I}}\right)$ (except rotations and vibrations) as calculated with our IAM-SCAR approach. Also given are the ICS for the Born dipole rotational excitations $\left(\sigma_{\text {rot }}\right)$ and the TCS that includes those cross sections $\left(\sigma_{\mathrm{T}+\mathrm{rot}}\right)$.

\begin{tabular}{lccccc}
\hline \hline Energy $(\mathrm{eV})$ & $\sigma_{\mathrm{E}}\left(10^{-20} \mathrm{~m}^{2}\right)$ & $\sigma_{\mathrm{I}}\left(10^{-20} \mathrm{~m}^{2}\right)$ & $\sigma_{\mathrm{T}}\left(10^{-20} \mathrm{~m}^{2}\right)$ & $\sigma_{\text {rot }}\left(10^{-20} \mathrm{~m}^{2}\right)$ & $\sigma_{\mathrm{T}+\mathrm{rot}}\left(10^{-20} \mathrm{~m}^{2}\right)$ \\
\hline 1 & 68.33 & 0 & 68.33 & 114.53 & 182.86 \\
1.5 & 53.49 & 0 & 53.49 & 80.09 & 133.57 \\
2 & 47.60 & 0 & 47.60 & 62.17 & 109.77 \\
3 & 42.00 & 0 & 42.00 & 43.12 & 85.13 \\
4 & 37.52 & 0 & 37.52 & 33.32 & 70.85 \\
5 & 34.16 & 0.11 & 34.27 & 27.34 & 61.61 \\
7 & 28.56 & 1.23 & 29.80 & 20.19 & 49.85 \\
10 & 15.15 & 21.17 & 36.32 & 14.67 & 50.97 \\
15 & 11.79 & 26.83 & 38.62 & 10.14 & 48.72 \\
20 & 11.06 & 27.58 & 38.64 & 7.70 & 46.48 \\
30 & 10.45 & 26.63 & 37.08 & 5.29 & 42.28 \\
40 & 9.83 & 25.20 & 35.03 & 4.20 & 39.20 \\
50 & 9.24 & 23.86 & 33.10 & 3.36 & 36.40 \\
70 & 8.09 & 21.65 & 29.74 & 2.46 & 32.20 \\
100 & 6.78 & 19.13 & 25.90 & 1.79 & 27.69 \\
150 & 5.29 & 16.16 & 21.45 & 1.23 & 22.65 \\
200 & 4.31 & 14.03 & 18.34 & 0.92 & 19.29 \\
300 & 3.19 & 11.20 & 14.39 & 0.64 & 15.04 \\
400 & 2.55 & 9.35 & 11.90 & 0.50 & 12.41 \\
500 & 2.13 & 8.06 & 10.20 & 0.39 & 10.59 \\
700 & 1.63 & 6.36 & 7.98 & 0.28 & 8.26 \\
1000 & 1.22 & 4.87 & 6.09 & 0.20 & 6.27 \\
\hline \hline
\end{tabular}

In Figure 3 we show the present measured TCS, both uncorrected and corrected for the forward angle scattering effect, as well as our IAM-SCAR TCS computed with and without the Born dipole rotational excitations. We note that a legitimate comparison between our theory and experiment can probably only be made when comparing our measured TCS corrected for forward scattering against the results of our computations that include the rotational excitations. This is because the rotational excitations cause the DCS to be strongly forward peaked (see Figure 2) and, as the present experiment misses part of the forward angle scattering, our measurements are unlikely to account for this effect on the TCS. In making the correction, we find only a qualitative level of accord between our corrected experimental TCS and the theoretical TCS with the rotational excitations. That computation lies largely above the corrected measured TCS, until about $\sim 60 \mathrm{eV}$ when it appears to become smaller in magnitude. Note that we do not a priori expect our computations to be accurate at the lower energies, as it is at those energies where the SCAR approach starts to fail (see Sec. III). Therefore, the disagreement between our theory and the corrected experimental results suggests that further development of the present formalism might be required and/or that the correction to our measured TCS for the forward angle scattering effect is underestimated at the lower energies. As the missing angle in our experiment is known with little uncertainty, the second explanation would imply that our theoretical DCS could be more accurate, which also suggests that an improvement in our positron scattering model is warranted.

We also observe in Figure 3 the important contribution of the rotational excitations to the calculated TCS, particu- larly at the lower energies (see also Table V). We note that the present measured TCS uncorrected for forward scattering lies above our calculated results including the rotational excitations above $100 \mathrm{eV}$, a result which we had not anticipated. Above $100 \mathrm{eV}$, where the effects of Ps formation (see Figure 4) in positron scattering and exchange in electron scattering "turn off," we would expect the electron and positron TCS to be largely identical. This is precisely what we see if we compare our measured positron TCS with the calculated electron TCS of Milosavljevic et al. ${ }^{14}$ (also shown in Figure 3). On the other hand, our calculated positron TCS remains uniformly lower in magnitude when compared to the corresponding electron TCS. This latter observation additionally suggests further development in our positron scattering model is required. Finally, we note a "dip" in our calculated TCS that seems to correspond to the opening of the first inelastic channel (see Sec. IV B), as can be clearly inferred from Table V. As this "dip" is not observed in the Trento TCS data ${ }^{11}$ nor in our measured TCS, we can only conclude that it is an artefact of the present computation.

Also shown in Figure 3 are the TCS measured earlier at the University of Trento. ${ }^{11}$ Those data are uncorrected for the forward angle scattering effect, so that a direct comparison against our preferred results, the present theory including the rotational excitations and the current measurements corrected for forward scattering, is not possible. Nevertheless we see that both our corrected experimental TCS and calculated TCS with rotations are higher in magnitude compared to the Trento data set at all common energies, which is precisely what we had anticipated. If we now compare the Trento data 
set to the current uncorrected experimental TCS, we find very good agreement with those previous results at energies below $\sim 3 \mathrm{eV}$, often to within their overall error bars. However, the data of Zecca et al. ${ }^{11}$ diverge from our experimental results at $\sim 3 \mathrm{eV}$ and above. On comparing the Trento data with our calculated (without rotations) TCS, we find quite good accord up to about $7 \mathrm{eV}$. The major difference between the Trento data and the present is that their TCS continues to decrease in magnitude towards the higher energies, while the current results show a plateau in magnitude through the onset of Ps formation and direct ionization. This mirrors the observation we made in our earlier investigation on $\mathrm{THF}^{9}{ }^{9}$ when we similarly compared our experimental and theoretical results to those of the Trento group. ${ }^{15}$ As in that previous study, we are a little surprised by this observed disagreement, as the investigations on the noble gases ${ }^{26,46-50}$ had revealed a quite satisfactory level of accord between the uncorrected (for forward scattering) TCS measured by the two groups. The "missing angle" affecting the present measurements (Table II) appears to be comparable to the angular discrimination of the Trento apparatus, ${ }^{51}$ except perhaps at higher energies. Therefore, we would anticipate the correction to the TCS for the forward angle scattering effect to be comparable between the two data sets, although we note that in the case of a polar molecule such as 3H-THF (which has very forward peaked elastic DCS, see Figure 2) any small difference in the angular discriminations might make a significant difference in terms of the missing fraction of forward scattering. The purity of the $3 \mathrm{H}-\mathrm{THF}$ sample used in the present study is the same as that used in Trento, so that a difference in the target sample composition is unlikely to be the source of the observed discrepancy. Although we do not have a quantitative explanation for this observed discrepancy at the moment, we point out that while the measurements at Trento were conducted at room temperature $\left(\sim 24 \pm 2{ }^{\circ} \mathrm{C}\right)$, the target vapor in the scattering cell was at $\sim 60^{\circ} \mathrm{C}$ in the current experiments. Note that in our geometry the target molecules thermalize with the scattering cell walls and, therefore, the scattering chamber temperature can be considered to be a good approximation of the target vapor temperature in the scattering region. Such a temperature difference might have caused the conformational distributions of the 3H-THF molecules to change between the two samples, so that the scattering might be affected in different ways. Of perhaps more significance is that the different sample temperatures of the two groups would lead to different initial rotational states and, given the energy resolution of their spectrometers, vibrational state distributions in their samples. This might well have an effect on the scattering in each case, perhaps in part contributing to the discrepancy between their TCS.

\section{B. Inelastic cross sections}

We plot the present experimental Ps formation cross section in Figure 4, together with our IAM-SCAR summed inelastic ICS (without rotations and vibrations, but including Ps formation). We also list those results in Tables IV and $\mathrm{V}$, respectively. These are the first inelastic cross sections to be reported for 3H-THF. We observe in Figure 4 that the Ps formation cross section starts rising sharply at around the known experimental threshold (see Table I) and then peaks at $\sim 12 \mathrm{eV}$, before it starts decreasing in magnitude as the energy increases. At its maximum, the Ps formation cross section constitutes about one third of the TCS. The Ps formation channel seems to become negligible only above $170 \mathrm{eV}$, as the cross section approaches zero magnitude. However, above about $100 \mathrm{eV}$, it is relatively small as we expected.

In Figure 4 the shape of our computed summed inelastic ICS resembles that of the present Ps formation cross section, although the former is larger in magnitude than the latter and its onset occurs at a slightly higher energy. In fact, the summed inelastic ICS appears to rise markedly from $\sim 7 \mathrm{eV}$ and reaches a maximum at $20 \mathrm{eV}$ before it starts decreasing in magnitude at the higher energies. The theoretical onset of the lowest inelastic process (except rotations and vibrations), at about this energy, is somewhat in disagreement with the opening of the Ps formation cross section, that we observe in our experimental data, and also with the known Ps threshold energy (see Table I). We have observed this same kind of discrepancy in our work on $\mathrm{THF}^{9}$ and, as we argued in that paper, this reflects an intrinsic limitation in our theoretical approach.

\section{Elastic differential cross sections}

The current elastic DCS calculated with our IAM-SCAR approach with the Born dipole rotational excitations are plotted in Figure 2 and tabulated in Table III at selected energies between 1 and $50 \mathrm{eV}$. These results represent the first theoretical elastic DCS for 3H-THF. The dominant feature in Figure 2 is how the DCS, at all energies, dramatically rise in magnitude as the scattered positron angle goes from $180^{\circ}$ towards $0^{\circ}$. Furthermore, those DCS become progressively more forward peaked as the incident positron energy becomes smaller. This is consistent with the strongly polar nature of $3 \mathrm{H}-\mathrm{THF}$ and with our finding in Sec. IV A of the enhanced scattering in the TCS towards the lower energies that is due to the predominance of the long-range polarization effects.

\section{Comparison between the cross sections for THF and 3H-THF}

In Figure 5 we now compare our (uncorrected) measured and calculated (without the rotational excitations) TCS for positron collisions with $3 \mathrm{H}-\mathrm{THF}$, with those from our previous investigation on the parent molecule THF. ${ }^{9}$ We note here that the missing angle (and the resulting correction) for the present experiment is very similar to that which affected the measurements on THF, ${ }^{9}$ so that a valid comparison between their uncorrected TCS can be made. The experimental TCS are consistent in both their energy dependence and magnitude, to within the combined error bars, throughout all of the common energies, except perhaps at a few of the higher energies where only a very slight difference is observed. This observation is in contrast with the conclusions of Zecca et al. ${ }^{11}$ who instead found similarities between their uncorrected TCS for THF and $3 \mathrm{H}-\mathrm{THF}$ only in the limited energy range of 
1.6-3.1 eV. Note, however, that the angular discrimination is not exactly the same for each experiment, so that it is difficult to draw any definitive conclusions in respect to this observation. It is also worth recalling here that while the present experiment on $3 \mathrm{H}-\mathrm{THF}$ was carried out with the target vapor held at $\sim 60{ }^{\circ} \mathrm{C}$, our previous measurements on THF were performed at room temperature $\left(\sim 24{ }^{\circ} \mathrm{C}\right)$. Notwithstanding this difference, the agreement we find between our measured TCS for these two species might reflect the similarity in their physical properties (see Table I). In fact, it is apparent from Table I that the anisotropic parameters of both THF and the global minimum conformer of $3 \mathrm{H}$-THF are very similar in value. In addition, although not shown, the geometric values (i.e., bond lengths and bond angles) of these species are also similar, suggesting that the molecular diameters of the species will be roughly equal. Moreover, the Ps formation threshold energies for these two targets are also very close to each other (again, see Table I). Under such circumstances one would, therefore, anticipate the energy dependence and magnitude of the TCS for these two molecules to be also similar, at least below the Ps formation threshold, which is exactly what we observe in Figure 5. We note that Vizcaino et al. ${ }^{13}$ also found little difference in their electron-impact elastic DCS measurements on $3 \mathrm{H}-\mathrm{THF}$ when compared to the corresponding results for THF. However, they also observed that despite the similarity in the shape and magnitude of their cross sections for both $3 \mathrm{H}-\mathrm{THF}$ and THF, their SMC calculation suggested that 3H-THF has a slightly larger cross-section than THF, whereas the experimental data seemed to show the opposite, particularly at the more forward and backward angles.

With respect to our calculated TCS (without the rotations), we see in Figure 5 that our 3H-THF results are, in general, larger in magnitude than those for THF: this difference is small at $1 \mathrm{eV}$ but becomes somewhat larger at the higher energies. We also find this trend when we compare our calculated summed inelastic ICS for these two related compounds in Figure 4. From a semi-classical perspective this may just reflect the fact that $3 \mathrm{H}-\mathrm{THF}$ is slightly larger in size than THF (3H-THF possesses an additional oxygen atom), but can also be explained in terms of the slightly bigger dipole moment and polarizability of the 1 st conformer of $3 \mathrm{H}-\mathrm{THF}$ (see Table I).

Figure 4 also shows the comparison between the present Ps formation cross section for $3 \mathrm{H}-\mathrm{THF}$ and that measured earlier for THF. ${ }^{9}$ The cross sections are essentially indistinguishable within the errors on the measurements. Above $90 \mathrm{eV}$ the cross sections for $3 \mathrm{H}-\mathrm{THF}$ appear to become slightly larger in magnitude than those for THF, although given the overall errors it is difficult to draw strong conclusions in the comparison between those data.

The qualitative and quantitative similarities observed in the present experimental and theoretical TCS and Ps formation cross section results for the related molecules THF and $3 \mathrm{H}-\mathrm{THF}$ seem to suggest that positron scattering from $3 \mathrm{H}-$ THF might be predominantly governed by its most energetically stable conformer, as this possesses the most similar properties to those of THF. This, of course, might lead us to speculate that in our $3 \mathrm{H}-\mathrm{THF}$ sample the conformer population distribution shows a significantly higher fraction of the most energetically stable conformer. However such a scenario is counter-intuitive, suggesting a more subtle interpretation for understanding why the present THF and 3H-THF cross sections are so similar and also why at the TCS level this observation is contrary to that found by Zecca et al. ${ }^{11}$ might be needed. For instance, some of the physico-chemical properties in Table I are determined through quantum chemical calculations employing model basis sets of states to represent the 3H-THF molecule. While the B3LYP/TZVP model chemistry has been shown to be quite robust in describing results from electron momentum spectroscopy studies ${ }^{52}$ and past studies on geometric and anisotropic parameters in biomolecules, ${ }^{53}$ it is well known that calculated physico-chemical properties do exhibit a dependence on the model chemistry employed. Thus, testing the robustness of the data in Table I, to changes in model chemistry, might be a useful first step to better understanding this issue.

\section{CONCLUSIONS}

In this paper we reported on a joint experimental and theoretical investigation into positron scattering from $3 \mathrm{H}-\mathrm{THF}$. We presented new measured TCS and original Ps formation cross sections for energies between 1 and $190 \mathrm{eV}$. In addition, we calculated original TCS, elastic and inelastic ICS, as well as some elastic DCS, with our IAM-SCAR theory at energies from 1 to $1000 \mathrm{eV}$. A qualitative level of accord was found between the present measured and computed TCS at all common energies. A comparison with the only earlier TCS measurement of the Trento group, ${ }^{11}$ revealed quite good agreement with our experimental data below the Ps formation threshold and with the present theoretical results below $\sim 7 \mathrm{eV}$. We also compared our measured and calculated TCS, Ps formation cross section and summed inelastic ICS for $3 \mathrm{H}-$ THF to our previous corresponding results for the structurally related species THF. ${ }^{9}$ This comparison highlighted how similar the cross sections for these two targets are to each other, an observation which is in contrast with that made earlier at the TCS level by Zecca et al. ${ }^{11}$

Notwithstanding the fair level of accord we noted above between our present measured and calculated TCS, one of the important findings of this study is that there is clearly further development required in our positron scattering model. This is probably not all that surprising given the complexity required for a quantitative theoretical description of positronmolecule scattering, particularly for complex molecular targets. Nonetheless, in spite of those complexities, we continue to develop our approach.

\section{ACKNOWLEDGMENTS}

The authors acknowledge the Australian Research Council (ARC) Centres of Excellence Program for funding. C.M. is also grateful to the ARC for financial support under the Australian Postdoctoral Fellowship program. G.G. and F.B. would like to acknowledge the Spanish Ministerio de Economía y Productividad (Project FIS2009-10245) and the European Science Foundation (COST Action MP1002 - Nano-IBCT) for financial support. L.C. would like to thank Darryl Jones 
for useful discussions. Finally we all thank the technical staff at the ANU, especially Stephen Battiston and Ross Tranter, for their invaluable input.

${ }^{1}$ B. Boudaiffa, P. Cloutier, D. Hunting, M. A. Huels, and L. Sanche, Science 287, 1658 (2000).

${ }^{2}$ G. García Gómez-Tejedor and M. C. Fuss, Radiation Damage in Biomolecular Systems (Springer, Berlin, 2012).

${ }^{3}$ S. Ptasińska, S. Denifl, P. Scheier, and T. Märk, J. Chem. Phys. 120, 8505 (2004).

${ }^{4}$ H. Abdoul-Carime, S. Gohlke, and E. Illenberger, Phys. Rev. Lett. 92, 168103 (2004)

${ }^{5}$ D. Bouchiha, J. D. Gorfinkiel, L. G. Caron, and L. Sanche, J. Phys. B 40, 1259 (2007)

${ }^{6}$ A. Giardini, D. Catone, S. Stranges, M. Satta, M. Taccani, S. Piccirillo, S. Turchini, N. Zema, G. Contini, T. Prospari, P. Decleva, D. DiTommaso, G. Fronzoni, and M. Stener, Chem. Phys. Chem. 6, 1164 (2005).

${ }^{7}$ X. Pan, P. Cloutier, D. Hunting, and L. Sanche, Phys. Rev. Lett. 90, 208102 (2003)

${ }^{8}$ F. Martin, P. D. Burrow, Z. Cai, P. Cloutier, D. Hunting, and L. Sanche, Phys. Rev. Lett. 93, 068101 (2004).

${ }^{9}$ L. Chiari, E. Anderson, W. Tattersall, J. R. Machacek, P. Palihawadana, C. Makochekanwa, J. P. Sullivan, G. García, F. Blanco, R. P. McEachran, M. J. Brunger, and S. J. Buckman, J. Chem. Phys. 138, 074301 (2013).

${ }^{10}$ D. Antic, L. Parenteau, and L. Sanche, J. Phys. Chem. B 104, 4711 (2000).

${ }^{11}$ A. Zecca, L. Chiari, A. Sarkar, and M. J. Brunger, J. Phys. B 41, 085201 (2008).

${ }^{12}$ P. Możejko and L. Sanche, Radiat. Phys. Chem. 73, 77 (2005).

${ }^{13}$ V. Vizcaino, J. Roberts, J. P. Sullivan, M. J. Brunger, S. J. Buckman, C. Winstead, and V. McKoy, New J. Phys. 10, 053002 (2008).

${ }^{14}$ A. R. Milosavljević, F. Blanco, J. B. Maljković, D Šević, G. García, and B. P. Marinković, New J. Phys. 10, 103005 (2008).

${ }^{15}$ A. Zecca, C. Perazzolli, and M. J. Brunger, J. Phys. B 38, 2079 (2005).

${ }^{16} \mathrm{See} \quad \mathrm{http}: / / \mathrm{www} . \mathrm{chemspider.com/Chemical-Structure.9566}$.html?rid= 06c0836d-3cb2-4cdc-b092-e85a3003d928 for a summary of some of the important physico-chemical data for 3-hydroxy-tetrahydrofuran.

${ }^{17}$ L. Chiari, A. Zecca, S. Girardi, A. Defant, F. Wang, X. G. Ma, M. V. Perkins, and M. J. Brunger, Phys. Rev. A 85, 052711 (2012).

${ }^{18}$ J. P. Sullivan, A. Jones, P. Caradonna, C. Makochekanwa, and S. J. Buckman, Rev. Sci. Intrum. 79, 113105 (2008).

${ }^{19}$ M. Kimura, O. Sueoka, A. Hamada, and Y. Itikawa, Adv. Chem. Phys. 111, 537 (2000).

${ }^{20}$ A. Zecca, N. Moser, C. Perazzolli, A. Salemi, and M. J. Brunger, Phys. Rev. A 76, 022708 (2007).

${ }^{21}$ L. Chiari, M. J. Brunger, and A. Zecca, Radiation Damage in Biomolecular Systems, edited by G. G. Gómez-Tejedor and M. C. Fuss (Springer, Berlin, 2012), p. 155.

${ }^{22}$ S. J. Gilbert, C. Kurz, R. G. Greaves, and C. M. Surko, Appl. Phys. Lett. 70, 1944 (1997).

${ }^{23}$ T. Takaishi and Y. Sensui, Trans. Faraday Soc. 59, 2503 (1963).

${ }^{24}$ M. Dampc, A. R. Milosavljevic, I. Linert, B. P. Marinković, and M. Zubek, Phys. Rev. A 75, 042710 (2007)

${ }^{25}$ J. P. Sullivan, S. J. Gilbert, J. P. Marler, R. G. Greaves, S. J. Buckman, and C. M. Surko, Phys. Rev. A 66, 042708 (2002).

${ }^{26}$ A. C. L. Jones, C. Makochekanwa, P. Caradonna, D. S. Slaughter, J. R. Machachek, R. P. McEachran, J. P. Sullivan, S. J. Buckman, A. D. Stauffer, I. Bray, and D. V. Fursa, Phys. Rev. A 83, 032701 (2011).
${ }^{27}$ P. Papp, P. Mach, J. Urban, and Š. Matejčík, Facta Univ. Ser. Phys. Chem. Tech. 6, 127 (2008).

${ }^{28}$ J. P. Sullivan, C. Makochekanwa, A. Jones, P. Caradonna, D. S. Slaughter, J. Machacek, R. P. McEachran, D. W. Mueller, and S. J. Buckman, J. Phys. B 44, 035201 (2011).

${ }^{29}$ A. Hamada and O. Sueoka, J. Phys. B 27, 5055 (1994).

${ }^{30}$ C. Makochekanwa, A. Banković, W. Tattersall, A. Jones, P. Caradonna, D. S. Slaughter, K. Nixon, M. J. Brunger, Z. Petrović, J. P. Sullivan, and S. J. Buckman, New J. Phys. 11, 103036 (2009).

${ }^{31}$ F. Blanco and G. García, Phys. Lett. A 295, 178 (2002).

${ }^{32}$ F. Blanco and G. García, Phys. Rev. A 67, 022701 (2003).

${ }^{33}$ F. Blanco and G. García, Phys. Lett. A 317, 458 (2003).

${ }^{34}$ F. Blanco and G. García, Phys. Lett. A 330, 230 (2004).

${ }^{35}$ R. P. McEachran, J. P. Sullivan, S. J. Buckman, M. J. Brunger, M. C. Fuss, A. Muñoz, F. Blanco, R. D. White, Z. Lj. Petrović, P Limão-Vieira, and G. García, J. Phys. B 45, 045207 (2012).

${ }^{36}$ L. Chiari, A. Zecca, S. Girardi, E. Trainotti, G. García, F. Blanco, R. P. McEachran, and M. J. Brunger, J. Phys. B 45, 215206 (2012).

${ }^{37}$ M. Charlton and J. W. Humberston, Positron Physics (Cambridge University Press, Cambridge, 2001).

${ }^{38}$ D. D. Reid and J. M. Wadehra, J. Phys. B 29, L127 (1996).

${ }^{39}$ R. P. McEachran, D. L. Morgan, A. G. Ryman, and A. D. Stauffer, J. Phys. B 10, 663 (1977).

${ }^{40}$ T. M. Miller and B. Bederson, Adv. At. Mol. Phys. 13, 1 (1977).

${ }^{41}$ E.-A. Reinsch and W. Meyer, Phys. Rev. A 18, 1793 (1978).

${ }^{42}$ A. K. Bhatia, Positron Interactions with Atoms and Ions (NASA/Goddard Space Flight Center, Maryland, 2012), see http://ntrs.nasa.gov/archive/ nasa/casi.ntrs.nasa.gov/20120011692_2012010939.pdf.

${ }^{43}$ F. Blanco and G. García, J. Phys. B 42, 145203 (2009).

${ }^{44}$ A. Jain, J. Phys. B 21, 905 (1988).

${ }^{45}$ A. S. Dickinson, J. Phys. B 10, 967 (1977).

${ }^{46}$ C. Makochekanwa, J. R. Machacek, A. C. L. Jones, P. Caradonna, D. S. Slaughter, R. P. McEachran, J. P. Sullivan, S. J. Buckman, S. Bellm, B. Lohmann, D. V. Fursa, I. Bray, D. W. Mueller, A. D. Stauffer, and M. Hoshino, Phys. Rev. A 83, 032721 (2011).

${ }^{47}$ A. Zecca, L. Chiari, E. Trainotti, D. V. Fursa, I. Bray, A. Sarkar, S. Chattopadhyay, K. Ratnavelu, and M. J. Brunger, J. Phys. B 45, 015203 (2012).

${ }^{48}$ A. Zecca, L. Chiari, E. Trainotti, D. V. Fursa, I. Bray, and M. J. Brunger, Eur. Phys. J. D 64, 317 (2011).

${ }^{49}$ J. R. Machacek, C. Makochekanwa, A. C. L. Jones, P. Caradonna, D. S. Slaughter, R. P. McEachran, J. P. Sullivan, S. J. Buckman, S. Bellm, B. Lohmann, D. V. Fursa, I. Bray, D. W. Mueller, and A. D. Stauffer, New J. Phys. 13, 125004 (2011).

${ }^{50}$ A. Zecca, L. Chiari, E. Trainotti, and M. J. Brunger, J. Phys. B 45, 085203 (2012).

${ }^{51}$ A. Zecca, L. Chiari, A. Sarkar, and M. J. Brunger, New J. Phys. 13, 115001 (2011).

${ }^{52}$ M. J. Brunger and W. Adcock, J. Chem. Soc., Perkin Trans. 2, 1 (2002).

${ }^{53}$ D. B. Jones, F. Wang, D. A. Winkler, and M. J. Brunger, Biophys. Chem. 121, 105 (2006).

${ }^{54}$ K. Kimura, S. Katsumata, Y. Achiba, T. Yamazaki, and S. Iwata, Handbook of He I Photoelectron Spectra of Fundamental Organic Molecules (Japan Chemical Societies Press, Tokyo, 1981).

${ }^{55}$ ChemBio3D Ultra, version 12.0, ChemBioOffice 2010, CambridgeSoft Corporation, Cambridge, MA, USA, 2010, see http://www.cambridgesoft. com. 